\title{
La Autoeficacia en el Cuidado de la Salud en la Predicción de la Satisfacción con la Vida
}

\author{
Self-Efficacy in Health Care in Prediction of Life Satisfaction
}

\author{
Luis H. Blanco Ornelas ${ }^{1}$, Ana C. Díaz-Leal ${ }^{2}$, Martha Ornelas Contreras ${ }^{3}$, Fernando Mondaca \\ Fernández ${ }^{4}$ y Natalia Solano Pinto ${ }^{5}$
}

\begin{abstract}
Resumen
Introducción y objetivos: En este estudio se han obtenido los datos psicométricos de los instrumentos de evaluación: Escala de autoeficacia en el cuidado de la alimentación y de la salud; Escala de satisfacción con la vida. Por otra parte se ha examinado un modelo predictivo de la satisfacción con la vida a través de los componentes de la autoeficacia. Método: Muestra, 279 jóvenes mujeres (17-28 años) procedentes de la Universidad Autónoma de Yucatán, México. Resultados: Los datos psicométricos de ambos cuestionarios confirman la propuesta factorial original. En el modelo predictivo de la satisfacción con la vida se ha encontrado una relación directa entre la satisfacción con la vida y la autoeficacia en el afrontamiento de problemas. Conclusiones: Los factores autoeficacia en el cuidado de la alimentación y afrontamiento de problemas adquieren relevancia en la explicación de la satisfacción con la vida.
\end{abstract}

Palabras clave: autoeficacia, satisfacción con la vida, afrontamiento de problemas

\begin{abstract}
Introduction and objectives: In this study, psychometric characteristics of two questionnaires designed to measure life satisfaction and specific diet-health perceived self- efficacy are discussed. On the other hand, a predictive model of life satisfaction is proposed, considering both self- efficacy components. Method: The sample included 279 women aged 17 to 28 years, studying in the Universidad Autónoma of Yucatán, México. Results: Psychometric properties related to both questionnaires confirm the original factorial proposal. The predictive model for life satisfaction shows a direct relationship between this variable and perceived coping self- efficacy. Conclusions: The self-efficacy in the care of diet and coping with problems factors acquire relevance in the explanation of life satisfaction.
\end{abstract}

Keywords: self-efficacy, life satisfaction, coping

Financión: Grupo de Investigación Mirada Crítica (Salud, Educación y Sociedad), financiado por la Universidad de Castilla La Mancha y cofinanciado por la Unión Europea a través del Fondo Europeo de Desarrollo Regional.

\footnotetext{
1 Médico. Residente de la especialidad de Medicina Interna. Unidad Hospitalaria "Fray Antonio Alcalde", Universidad de Guadalajara, México. Unidad Hospitalaria "Fray Antonio Alcalde" (Civil Viejo). Calle Hospital 278, Col. El Retiro Guadalajara, Jalisco, México. Tel.: 3339424400. Correo: mdluishblanco01@gmail.com

${ }_{2}$ Médico. Residente de la especialidad de Medicina Interna. Unidad Hospitalaria "Fray Antonio Alcalde", Universidad de Guadalajara, México. Unidad Hospitalaria "Fray Antonio Alcalde" (Civil Viejo). Calle Hospital 278, Col. El Retiro Guadalajara, Jalisco, México. Tel.: 3339424400. Correo: anacitlalli@gmail.com

${ }^{3}$ Doctora en Actividad Física y Salud. Profesora tiempo completo Facultad de Ciencias de la Cultura Física. Universidad Autónoma de Chihuahua. Facultad de Ciencias de la Cultura Física, Universidad Autónoma de Chihuahua. Cd. Universitaria, Apdo postal $2-$ 1585, Chihuahua, Chih. México. Tel.: 6141589900. Correo: mornelas@uach.mx

${ }^{4}$ C. Dr. en Educación. Profesor de medio tiempo. Facultad de Ciencias de la Cultura Física, Universidad Autónoma de Chihuahu, México.

${ }^{5}$ Doctora en Psicología. Contratada Doctora. Universidad Castilla La Mancha, España. Fábrica de Armas. Campus tecnológico. 45071, Toledo, España. Tel.: 925268800 ext: 5937. Correo: natalia.solano@uclm.es (Correspondencia)
} 


\section{Introducción}

Diferentes instituciones realizan recomendaciones sobre las pautas adecuadas para cuidar la salud con la finalidad de prevenir el desarrollo de enfermedades y fomentar el bienestar (Ávila, Huertas, \& Tercedor, 2016; Martín- Payo, SuárezÁlvarez, Ameva, Duaso, \& Álvarez, 2016). En este sentido, la Organización Mundial de la Salud (OMS, 2013) señala como una prioridad sanitaria la mejora del bienestar de la población fomentando la investigación de todos los procesos implicados.

Así, con el fin de promocionar eficazmente un estilo de vida saludable y activo, se ha investigado sobre los aspectos que pudieran facilitar la realización de dicho estilo (Amador, Montero, Beltrán- Carrillo, González- Cutre, \& Cervelló, 2017). Una de las variables estudiadas ha sido la autoeficacia, concepto ampliamente descrito desde la teoría de Bandura, donde se enfatiza que la autoeficacia es un aspecto clave, entre otros, para adquirir o mejorar, conductas saludables (Luszczynska \& Schwarzer, 2005). De esta forma, las creencias sobre la capacidad de cuidar la propia salud pueden predecir la realización de dicho autocuidado (Thrasher, et al, 2016). En esta línea, algunos autores han señalado a la autoeficacia como una variable predictora en la intención de ingerir alimentos bajos en calorías, frutas y verduras (Povey, Conner, Sparks, James, $\&$ Shepherd, 2000) mencionándola como uno de los elementos para fomentar el estilo de vida saludable (González- Cantero, et al, 2017), reducir el peso en personas adultas con sobrepeso (Annesi $\&$ Tennant, 2014) y mantenerlo en personas con normopeso (Teixeira, et al, 2010).

Además, los estudios señalan que la autoeficacia está ligada al bienestar (Maddux, 2005) y que dicha relación es fundamental para el mantenimiento del bienestar a lo largo de las distintas etapas del ciclo vital (Salanova, Martínez, Bresó, Llorens, \& Grau, 2005). Sin embargo, cabe destacar que en la adolescencia disminuye la práctica de actividad física siendo, la disminución, más acusada en la juventud (Práxeles, Sevil, Moreno, del Villar, \& GarcíaGonzález, 2016) fundamentalmente en las mujeres (Murillo, et al, 2015) debido a motivaciones estéticas (Roberts, Reeves, \& Ryrie, 2015) y una baja percepción de autoeficacia (Jose, Blizzard, Dwyer, Mckercher, \& Venn, 2011). Además, las mujeres encuentran presiones socioculturales para ejercer el rol de cuidadora alejada de la actividad física y el deporte (Castellanos \& Soriano, 2010).

En las investigaciones también aparece reflejada la relación entre conductas saludables y satisfacción con la vida (Gunnell, Crocker, Mack, Wilson, \& Zumbo, 2014). La valoración que realiza una persona de su trayectoria vital define al término satisfacción con la vida situado tradicionalmente como el componente cognitivo del bienestar (Diener, Lucas, \& Oishi, 2002). Según el modelo de Lyubomirsky (Lyubomirsky, Sheldon, \& Sihkade, 2005) la práctica de la actividad física llegaría a explicar el $40 \%$ de la satisfacción con la vida. Aunque existen autores que no han encontrado dicha asociación (Sollerhed, Apitzsch, Rastam, \& Ejlertsson, 2008) en la literatura científica se refleja el estudio de las variables mediadoras entre actividad física y satisfacción con la vida (Hecimovich, Peiffer, \& Harbaugh 2014; León, Núñez, Domínguez, \& Martín- Albo, 2013). También se ha relacionado el ejercicio físico con el consumo de tóxicos (Amezcua, Hernández, Rodríguez, \& Quesada, 2016), estableciendo una relación negativa entre el consumo de alcohol y tabaco y la realización de actividad física (Cerkez, Culjak, Zenic, Sekulic, \& Kondric, 2015), resaltando que el consumo de alcohol aumenta la probabilidad de consumir tabaco (Chacón, et al, 2016). Sin embargo, se resalta que la medición de conductas de salud es realizada a través de autoinforme o encuestas, siendo una limitación metodológica reflejada en parte de la literatura revisada (Ávila, et al, 2016; González- Cantero, et al, 2017; Práxeles, 2016).

Otra de las variables relacionadas con el bienestar ha sido las estrategias de afrontamiento (Sanjuán \& Ávila, 2016). El afrontamiento activo de problemas se encuentra relacionado con la autoeficacia (Reyes-Jarquin \& Hernández- Pozo, 2011; Vallejo-Sánchez \& Pérez-García, 2016) y con el bienestar emocional (Sanjuán \& Magallares, 2014). Sin embargo, en la revisión realizada no se han encontrado estudios que relacionen cómo interactúan las diferentes conductas saludables (alimentación equilibrada, realización de actividad física, evitación de tóxicos) con el afrontamiento de problemas. 
Aunque la práctica de comportamientos saludables, fundamentalmente la actividad física, está relacionado con el bienestar psicológico (Gunnell, et al, 2014) no se encuentra estudiado el papel de la autoeficacia. En este sentido, existe una literatura extensa sobre la autoeficacia en el ámbito académico (Oros, 2017), sin embargo, en la revisión realizada se observa escasa investigación en el ámbito de la salud, fundamentalmente sobre el papel de la autoeficacia en el cuidado de la salud en la predicción de la satisfacción con la vida. Por ello los objetivos de este trabajo han sido los siguientes:

(1) Evaluar las propiedades psicométricas de los cuestionarios Escala de Autoeficacia en el Cuidado de la Alimentación y Salud (Blanco, Ornelas, Viciana, \& Rodríguez-Villalobos, 2016) y la Escala de Satisfacción con la Vida (Atienza, Pons, Balaguer, \& García-Merita, 2000).

(2) Construir un modelo predictivo de satisfacción con la vida a partir de los factores de autoeficacia en la realización del ejercicio físico, cuidado de la alimentación, afrontamiento de problemas, evitación del consumo de tabaco y alcohol.

\section{Hipótesis}

Se ha propuesto un modelo inicial que integra las posibles relaciones entre la autoeficacia en el cuidado de la salud y la satisfacción con la vida (Figura 1). De dicho modelo se desprenden las hipótesis entre los factores que a continuación se enuncian.

H1: Autoeficacia en la evitación del consumo de tabaco ejerce un efecto indirecto sobre la satisfacción con la vida siendo las variables mediadoras: autoeficacia en la evitación del consumo de alcohol, afrontamiento de problemas y cuidado de la alimentación.

H2: La autoeficacia en la realización de ejercicio físico ejerce un efecto indirecto sobre la satisfacción con la vida siendo las variables mediadoras: autoeficacia en el cuidado de la alimentación y afrontamiento de problemas.

H3: La autoeficacia en la evitación del consumo de alcohol ejerce un efecto indirecto sobre la satisfacción con la vida siendo las variables mediadoras: la autoeficacia en el afrontamiento de problemas y cuidado de la alimentación.

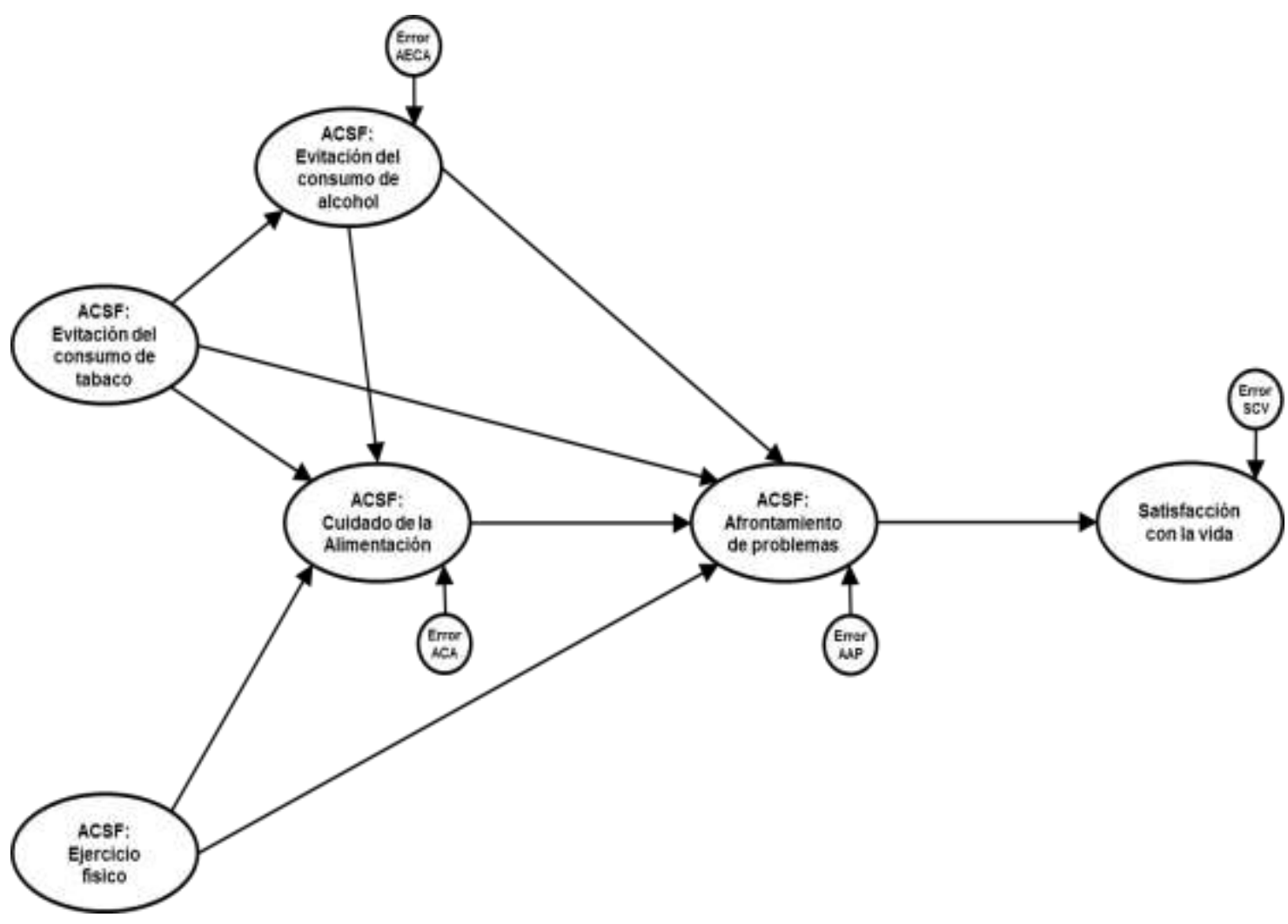

Figura 1. Modelo inicial propuesto para satisfacción con la vida en función de la autoeficacia en el cuidado de la alimentación y la salud física. Los indicadores de los factores no están representados por razones de simplicidad en la presentación 
H4: La autoeficacia en el cuidado de la alimentación ejerce un efecto indirecto sobre la satisfacción con la vida a través del factor afrontamiento de problemas.

H5: La autoeficacia en el afrontamiento de problemas ejerce un efecto directo sobre la satisfacción con la vida.

\section{Método}

\section{Participantes}

Participaron en el estudio 279 mujeres universitarias de la Facultad de Psicología (Universidad Autónoma de Yucatán, México), por tanto, la muestra fue por conveniencia mediante un método de muestreo no aleatorio La edad de las participantes fluctuó entre los 17 y 28 años $(\mathrm{M}=20.44$; DT=1.76).

\section{Instrumentos}

Escala de autoeficacia en el cuidado de la alimentación y salud física (EACASF) de J. R. Blanco et al. (2016). Evalúa la autoeficacia en la realización de conductas relacionadas con el cuidado de la salud. Consta de 28 ítems agrupados en cinco factores: 1. Cuidado de la alimentación (6 ítems); 2. Ejercicio físico (6 ítems); 3. Evitación del consumo de tabaco (5 ítems); 4. Evitación del consumo de alcohol (5 ítems); y 5. Afrontamiento de problemas (6 ítems). Se trata de una escala de tipo Likert de 11 puntos donde el participante responde si se siente capaz de realizar cada una de las conductas relacionadas con el cuidado de la salud ( $0=$ nada capaz; 10 absolutamente capaz).

Escala de satisfacción con la vida (Satisfaction With Life Scale, SWLS) de Diener, Emmons, Larsen y Griffin (1985), adaptada por Atienza et al., (2000). Evalúa, mediante 5 ítems, el juicio global que hacen las personas sobre la satisfacción con su propia vida. La adaptación de Atienza propone cinco opciones de respuesta aunque en este estudio se utilizó una escala líkert de 11 puntos $(0=$ completamente en desacuerdo; $10=$ completamente de acuerdo).

Se consideró que la escala líkert utilizada se adaptaba al sistema de calificación (0-10) que habitualmente se utiliza en el ámbito educativo, favoreciendo, de esta forma, la cumplimentación por parte de las participantes.

Los datos psicométricos da ambos instrumentos fueron objeto de estudio utilizándose solo los ítems que presentaban mejores resultados en los análisis factoriales confirmatorios.

\section{Procedimiento}

Este estudio, siguiendo el modelo de ecuaciones estructurales, pretende predecir la satisfacción con la vida a partir de la autoeficacia en el cuidado de la salud. Para ello, a través de una investigación cuantitativa de corte transversal se aplicaron dos cuestionarios. La investigación tuvo en cuenta las recomendaciones de la Declaración de Helsinki en relación a la privacidad, confidencialidad y consentimiento informado. Una vez obtenidos los permisos por parte de la Facultad de Psicología y de la Universidad de Yucatán (México); se informó a las estudiantes $\mathrm{y}$, las que decidieron voluntariamente participar, firmaron el consentimiento informado. En dicho consentimiento se detallaba las condiciones de la investigación recalcando el carácter voluntario y el tratamiento anónimo de sus datos. Después, se aplicaron los instrumentos de evaluación de forma grupal, en una sola sesión de 45 minutos en horario lectivo, por medio de un ordenador personal del Centro de Cálculo de la propia Facultad de Psicología. Al inicio de la sesión se hizo una pequeña introducción sobre la importancia de la investigación y de cómo acceder a los instrumentos. Se solicitó la máxima sinceridad y se garantizó la confidencialidad de los datos. Las instrucciones de cómo responder se encontraban en las primeras pantallas; antes del primer ítem de cada instrumento. Al término de la sesión se les agradeció su participación. Una vez aplicados los instrumentos se procedió a recopilar los resultados por medio del módulo generador de resultados del editor de escalas versión 2.0 (Blanco, et al, 2013). Por último, los resultados obtenidos se analizaron mediante el paquete SPSS 18.0 y AMOS 21.0.

Debido a las características muestrales y las franjas etarias deben considerarse las limitaciones en relación a la validez externa de los resultados. 


\section{Análisis de datos}

\section{Análisis de las propiedades psicométricas de los instrumentos}

Para comprobar la adecuación de la estructura factorial de los instrumentos con la muestra estudiada se realizaron análisis factoriales confirmatorios (AFCs) mediante el programa AMOS 21. El método de estimación empleado fue el de Máxima Verosimilitud. Se compararon los índices de ajuste de varios modelos con la finalidad de seleccionar el que mejor ajuste presentase. De esta forma, se siguieron las recomendaciones de Thompson (2004) en relación a los AFCs.

Por tanto, para ambos instrumentos de evaluación, se sometieron a comparación dos modelos de medida: el Modelo 1 (M1), modelo acorde a la distribución original propuesta por los autores de los cuestionarios y el Modelo 2 (M1b), que responde a la estructura factorial más adecuada tomando en cuenta a los índices de modificación y su justificación teórica; eliminando los ítems que no fueron suficientemente bien explicados por el modelo M1 correspondiente. Se consideraron los ítems que saturaban por encima de .70 siguiendo las recomendaciones que se realizan al respecto en ciencias sociales (Losada, Varela, \& Lévy, 2006).

Posteriormente, se calculó la fiabilidad de cada una de los factores de los mejores modelos obtenidos en los cuestionarios analizados, a través del Coeficiente Alpha de Cronbach.

\section{Análisis del modelo de ecuaciones estructurales para predecir la satisfacción con la vida a partir de la autoeficacia en el cuidado de la salud}

Antes de emplear el análisis de ecuaciones estructurales (SEM) se comprobó que se cumplían los supuestos subyacentes a esta técnica, especialmente los de normalidad y linealidad, para lo cual se analizaron los valores de asimetría y curtosis y los gráficos de dispersión matricial de las distintas variables contempladas en cada modelo.

Luego, a partir de la matriz de correlaciones, se utilizó SEM utilizando el método de estimación de máxima verosimilitud (ML). El ajuste de los modelos se comprobó a partir del Chi-cuadrado, el índice de bondad de ajuste (GFI), la raíz media cuadrática residual estandarizada (SRMR) y el error cuadrático medio de aproximación (RMSEA) como medidas absolutas de ajuste. El índice de bondad ajustado (AGFI), el Índice Tucker-Lewis (TLI) y el índice de bondad de ajuste comparativo (CFI) como medidas de ajuste incremental. La razón de Chi-cuadrado sobre los grados de libertad (CMIN/GL) y el Criterio de Información de Akaike (AIC) como medidas de ajuste de parsimonia. Para el GFI, AGFI, TLI y CFI se estableció como umbral de aceptación valores superiores a .90 , e inferiores a .08 para el RMSEA y el SRMR.

Posteriormente, se analizaron los efectos directos, indirectos y totales obtenidos entre las distintas variables contempladas en el modelo.

\section{Resultados}

Propiedades psicométricas de los instrumentos de evaluación

Escala de autoeficacia en el cuidado de la alimentación y salud física (EACASF). Los resultados globales del análisis factorial confirmatorio (GFI .844; RMSEA .066; SRMR .063; AGFI .810; TLI .926; CFI .926; CMIN/DF 2.196; AIC 877.206) para el modelo M1, modelo de cinco factores acorde a la distribución original de los ítems en el EACASF, indican que el modelo de medición es aceptable. El conjunto de los cinco factores del modelo M1 explican aproximadamente el $69 \%$ de la varianza. Sin embargo, cinco de los 28 ítems saturan por debajo de .70 en su dimensión prevista (ver Tabla 1). Respecto a la validez discriminante, se observan correlaciones moderadas entre los factores.

Los resultados globales del análisis factorial confirmatorio (GFI .970; RMSEA .018; SRMR .063; AGFI .810; TLI .917; CFI .998; CMIN/DF 1.085; AIC 113.017), del segundo modelo sometido a prueba (M1b) que corresponde a una estructura pentadimensional del cuestionario sin los ítems de más baja saturación, indican que este modelo de medición es mejor que el modelo anterior y que su ajuste es óptimo. Los cinco factores de este modelo explican en conjunto aproximadamente el $82 \%$ de la varianza. Por otro lado de acuerdo a los resultados de la Tabla 1; 
Tabla 1. Soluciones estandarizadas análisis factorial confirmatorio para autoeficacia en el cuidado salud

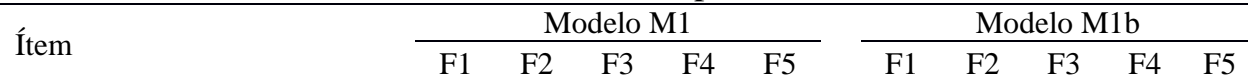

17 Realizar ejercicio físico durante cuando menos 30 minutos tres o más sesiones a la semana

18 Hacer ejercicio físico, a pesar de tener preocupaciones y .91 problemas

19 Hacer ejercicio físico, a pesar de sentirme deprimido

20 Hacer ejercicio físico, a pesar de sentirme tenso

21 Hacer ejercicio físico, a pesar de sentirme cansado

22 Hacer ejercicio físico, a pesar de estar ocupado

11 Resistirme a comer cuando estoy ansioso o nervioso

12 Resistirme a comer cuando estoy deprimido o experimento un fracaso

13 Resistirme a comer cuando hay mucha comida disponible

14 Resistirme a comer cuando otros me están presionando para hacerlo

15 Resistirme a comer alimentos con muchas calorías aun cuando me gusten mucho

16 Resistirme a comer a pesar de que otros se molesten si yo no como

23 Funcionar lo más normalmente posible cuando tengo problemas o contratiempos en mi vida

24 Que los problemas o contratiempos que tengo no afecten a mis emociones, relaciones $\mathrm{u}$ otras esferas de mi vida

25 Afrontar eficazmente problemas o contrariedades en mi vida

26 No sentirme tenso o ansioso cuando tengo problemas o contratiempos

27 Prestar atención a otras cosas cuando tengo problemas o contrariedades

28 Poner en marcha de manera efectiva todos los recursos que están a mi alcance para resolver un problema o contratiempos que tenga

1 Evitar el consumo de tabaco a pesar de las presiones de mis amigos

2 Controlarme y reducir mi consumo de tabaco

3 Resistir la tentación de consumir tabaco en exceso cuando estoy deprimido o experimento un fracaso

4 Resistirme a consumir tabaco cuando estoy ansioso o nervioso

5 Controlarme y no consumir nada de tabaco

6 Evitar el consumo de bebidas alcohólicas a pesar de las presiones de mis amigos

7 Controlarme y reducir mi consumo de alcohol

8 Resistir la tentación de beber en exceso cuando estoy deprimido o experimento un fracaso

9 Resistirme a consumir bebidas alcohólicas cuando estoy ansioso o nervioso

10 Controlarme y no beber nada de alcohol
Pesos Factoriales

.82

.83

.86

.79

.79

.67

.65

.78

.83

.64

.66

.73

.50

.77

.79

.81

.80

.78

.80

.69

.76

.70

.70

Correlaciones Factoriales

$$
\begin{array}{lcccc}
\text { F1 } & - & & & \\
\text { F2 } & .43^{*} & - & & \\
\text { F3 } & .42^{*} & .48^{*} & - & \\
\text { F4 } & .00 & .22^{*} & .18^{*} & - \\
\text { F5 } & .00 & .35^{*} & .35^{*} & .43^{*}
\end{array}
$$

.72

.76

$\begin{array}{ll}.96 & .98\end{array}$

.94

.93

.79

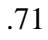

.92

.80

Nota. $* p<.05 ;$ F1=Ejercicio físico F2=Cuidado de la Alimentación F3=Afrontamiento de problemas F4=Evitación del consumo de tabaco F5=Evitación del consumo de alcohol

todos los ítems, con excepción del ítem 14, saturan igual o por encima de .70 en su dimensión prevista. Observándose además, correlaciones moderadas entre los dos factores evidenciando una adecuada validez discriminante entre ellos. 


\section{Escala de satisfacción con la vida (SWLS)}

Para el modelo 1, modelo unifactorial acorde a la distribución original de los ítems de la escala SWLS, el modelo de medición es óptimo según el análisis factorial confirmatorio (GFI .990; RMSEA .041; SRMR .020; AGFI .969; TLI .993; CFI .996; CMIN/DF 1.470; AIC 27.349). El modelo unifactorial del M1 explica aproximadamente el $66 \%$ de la varianza. Sin embargo, algunos de los ítems saturan por debajo de .70 (ver Tabla 2).

Tabla 2. Soluciones estandarizadas análisis factorial confirmatorio para el Modelo M1. Satisfacción con la vida

\begin{tabular}{lcc}
\hline \multicolumn{1}{c}{ Ítem } & \multicolumn{2}{c}{ Pesos Factoriales } \\
\cline { 2 - 3 } & $\begin{array}{c}\text { Modelo } \\
\text { M1 }\end{array}$ & $\begin{array}{c}\text { Modelo } \\
\text { M1b }\end{array}$ \\
\hline $\begin{array}{l}\text { 1. En la mayoría de los aspectos mi } \\
\text { vida es como yo quiero que sea }\end{array}$ & .77 & .77 \\
$\begin{array}{l}\text { 2. Las circunstancias de mi vida son } \\
\text { muy buenas }\end{array}$ & .74 & .74 \\
$\begin{array}{l}\text { 3. Estoy satisfecho con mi vida } \\
\text { 4. Hasta ahora he conseguido de la } \\
\text { vida las cosas que considero }\end{array}$ & .92 & .92 \\
$\begin{array}{l}\text { importantes } \\
\text { 5. Si pudiera vivir mi vida otra vez no } \\
\text { cambiaría casi nada }\end{array}$ & .67 & .67 \\
\hline
\end{tabular}

Los resultados globales del análisis factorial confirmatorio (GFI .997; RMSEA .001; SRMR .011; AGFI .984; TLI 1; CFI 1; CMIN/DF 0.901; AIC 17.802), del segundo modelo sometido a prueba (M1b) que corresponde a la estructura del Modelo 1 sin el ítem 5, indican que este modelo de medición es mejor que el modelo anterior y que su ajuste es óptimo explicando el modelo aproximadamente el $70 \%$ de la varianza. Por otro lado de acuerdo a los resultados de la tabla II; todos los ítems, salvo el ítem 4, saturan por encima de 70 en su dimensión prevista.

Respecto a la fiabilidad de los factores obtenidos (consistencia interna) en ambos cuestionarios, poseen valores por encima de .70 evidenciando una consistencia interna adecuada para este tipo de subescalas, particularmente si se considera el número reducido de ítems (ver Tabla 3).

Tabla 3. Coeficientes alfa para los factores obtenidos

\begin{tabular}{lc}
\hline \multicolumn{1}{c}{ Factor } & $\alpha$ \\
\hline Ejercicio físico & .882 \\
Cuidado de la Alimentación & .706 \\
Afrontamiento de problemas & .837 \\
Evitación del consumo de tabaco & .949 \\
Evitación del consumo de alcohol & .864 \\
Satisfacción con la vida & .830 \\
\hline
\end{tabular}

Modelo de ecuaciones estructurales para predecir la satisfacción con la vida a partir de la autoeficacia en el cuidado de la salud

Respecto a los valores de asimetría y curtosis, cuatro de las 15 variables muestran valores de asimetría y de curtosis que indican falta de normalidad univariada (ítems 3, 4, 8 y 9 del cuestionarios ACSF). Además, el índice multivariado de Mardia se encuentra por encima del valor 70, por lo que se puede inferir que no existe normalidad multivariada.

En relación al ajuste global de los modelos planteados, los resultados globales del análisis factorial confirmatorio para el modelo inicial propuesto indican que su ajuste es óptimo (ver tabla 4). Sin embargo, se realizó una reespecificación del modelo inicial eliminando la relación no significativa: evitación del consumo de tabaco - Afrontamiento de problemas (ver Figura 2).

Tras la eliminación de la vía (path) no significativa, el modelo final sigue presentando un ajuste óptimo (ver Tabla 4).

Como se observa en la Figura 2, tanto el modelo inicial propuesto como el modelo final explican aproximadamente el $22 \%$ de la varianza en la percepción de satisfacción con la vida (ver Figura 2).

Analizando individualmente los coeficientes de regresión para cada una de las vías propuestas en el modelo final (ver Figura 2), se observó que la totalidad de las relaciones propuestas obtienen significación cuando menos a un nivel $p<.05$.

Tal y como se muestra en la tabla 5 el efecto directo más elevado fue el que produce el factor afrontamiento de problemas en la satisfacción con la vida $(\beta=.47, p<.001)$, seguido del producido por evitación del consumo de tabaco sobre la evitación del consumo de alcohol ( $\beta=.37, p<.001)$ y el ejercicio físico sobre el cuidado de la alimentación $(\beta=.35, p<.001)$. De manera similar destaca el efecto del ejercicio físico sobre el afrontamiento de problemas $(\beta=.29, p<.001)$. Por otro lado, los resultados muestran que los factores ejercicio físico, evitación del consumo de tabaco y de alcohol ejercen un efecto indirecto significativo sobre la satisfacción con la vida a través de los factores cuidado de la alimentación y afrontamiento de problemas. Por su parte los factores ejercicio físico, evitación del consumo de 


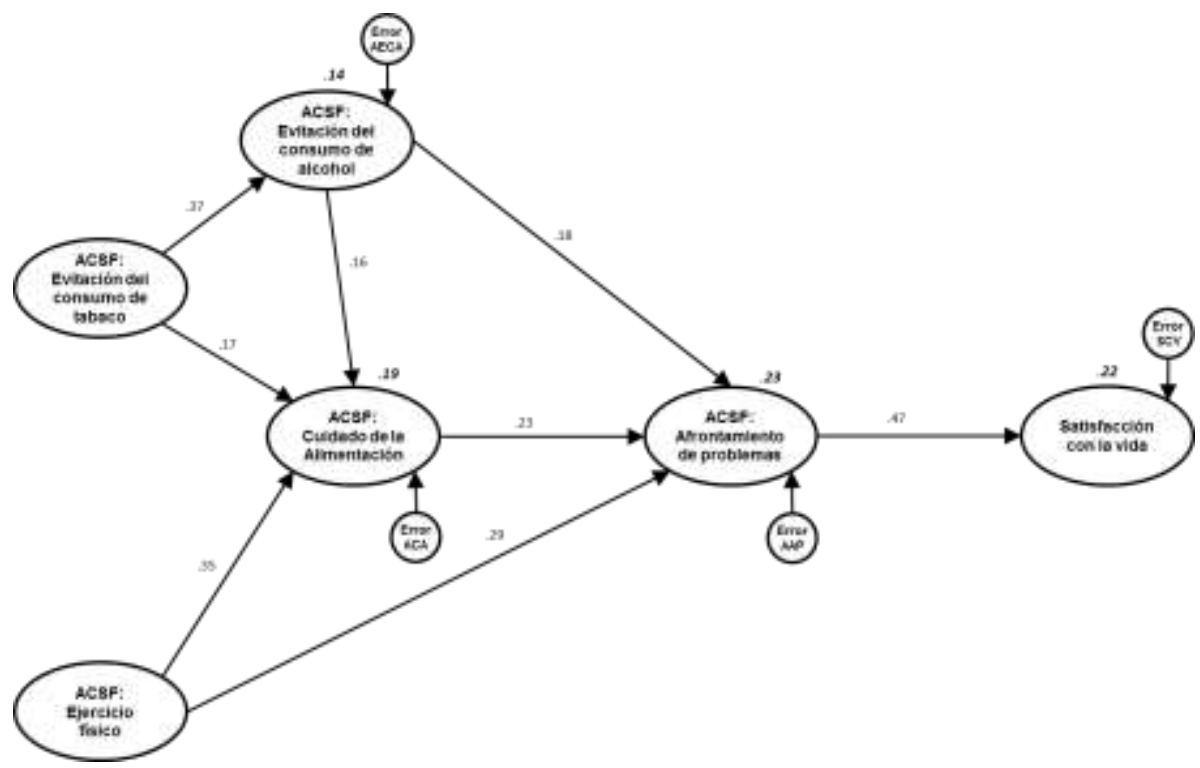

Figura 2. Solución estandarizada del modelo estructural final para satisfacción con la vida en función de la autoeficacia en el cuidado de la alimentación y la salud física. Todos los parámetros están estandarizados

Tabla 4. Índices absolutos, incrementales y de parsimonia de los modelos inicial y final satisfacción con la vida en función de la autoeficacia en el cuidado de la alimentación y la salud física

\begin{tabular}{|c|c|c|c|c|c|c|c|c|c|}
\hline \multirow[b]{2}{*}{ Modelo } & \multicolumn{4}{|c|}{ Índices absolutos } & \multicolumn{3}{|c|}{ Índices incrementales } & \multicolumn{2}{|c|}{ Índices de parsimonia } \\
\hline & $x^{2}$ & GFI & RMSEA & SRMR & AGFI & TLI & CFI & CMIN/DF & AIC \\
\hline Inicial & $133.952 *$ & .944 & .048 & .044 & .918 & .969 & .976 & 1.634 & 209.952 \\
\hline Final & $135.256^{*}$ & .944 & .048 & .047 & .918 & 969 & .976 & 1.630 & 209.256 \\
\hline
\end{tabular}

Nota. ${ }^{*} p<.05$; GFI=índice de bondad de ajuste; RMSEA=error cuadrático medio de aproximación; SRMR=raíz media cuadrática residual estandarizada; AGFI=índice de bondad ajustado; TLI=índice de Tucker-Lewis; CFI=índice de bondad de ajuste comparativo; $\mathrm{CMIN/DF=la} \mathrm{razón} \mathrm{de} \mathrm{Chi-cuadrado} \mathrm{sobre} \mathrm{los} \mathrm{grados} \mathrm{de} \mathrm{libertad;} \mathrm{AIC=criterio} \mathrm{de} \mathrm{información} \mathrm{de}$ Akaike.

Tabla 5. Efectos directos e indirectos estandarizados entre las variables consideradas en el modelo estructural final para satisfacción con la vida en función de la autoeficacia en el cuidado de la salud

\begin{tabular}{|c|c|c|c|c|c|c|}
\hline & & $\begin{array}{c}\text { evitación del } \\
\text { consumo de } \\
\text { tabaco }\end{array}$ & $\begin{array}{l}\text { ejercicio } \\
\text { físico }\end{array}$ & $\begin{array}{c}\text { evitación del } \\
\text { consumo de } \\
\text { alcohol }\end{array}$ & $\begin{array}{l}\text { cuidado de la } \\
\text { alimentación }\end{array}$ & $\begin{array}{l}\text { afrontamiento } \\
\text { de problemas }\end{array}$ \\
\hline \multirow{2}{*}{$\begin{array}{l}\text { evitación del consumo de } \\
\text { alcohol }\end{array}$} & Directo & .368 & & & & \\
\hline & Indirecto & & & & & \\
\hline \multirow{2}{*}{ cuidado de la alimentación } & Directo & .170 & .346 & .156 & & \\
\hline & Indirecto & .058 & & & & \\
\hline \multirow{2}{*}{ afrontamiento de problemas } & Directo & & .289 & .178 & .232 & \\
\hline & Indirecto & .118 & .080 & .036 & & \multirow{3}{*}{.469} \\
\hline \multirow{2}{*}{ satisfacción con la vida } & Directo & & & & & \\
\hline & Indirecto & .055 & .173 & .100 & .109 & \\
\hline
\end{tabular}

Nota. Variables Explicativas (primera columna), variables explicadas (primera fila)

\section{Discusión}

alcohol y cuidado de la salud física influyen indirectamente de forma significativa en la satisfacción con la vida a través del factor afrontamiento de problemas. Finalmente, se puede observar que los signos de los efectos directos corresponden a los esperados.
En el estudio realizado se ha obtenido un modelo predictivo de la satisfacción con la vida a través de las dimensiones de la autoeficacia en el cuidado de la salud, así como la relación entre las propias dimensiones. En dicho modelo se han establecido relaciones directas e indirectas con los 
aspectos de autoeficacia en el cuidado de la salud evaluados mediante el cuestionario EACASF. Dada la importancia de la autoeficacia en la adquisición de hábitos saludables (Luszczynskay $\&$ Schwarzer, 2005) y su capacidad predictiva en la realización de conductas de autocuidado de la salud (Thrasher, et al, 2016), se ha considerado fundamental asegurar que el instrumento de evaluación es válido y fiable en la muestra evaluada. En este sentido, los análisis factoriales confirmatorios para el cuestionario de autoeficacia en el cuidado de la alimentación y la salud física (EACASF) muestran que una estructura pentafactorial es viable y adecuada. La estructura de cinco factores (ejercicio físico, cuidado de la alimentación, afrontamiento de problemas, evitación del consumo de tabaco y evitación del consumo de alcohol), atendiendo a criterios estadísticos y sustantivos, ha mostrado adecuados indicadores de ajuste de fiabilidad y validez, además de evidenciar una fiabilidad adecuada en todos los factores.

En la misma línea, y dada la importancia del estudio en la satisfacción con la vida como componente cognitivo del bienestar, (OMS, 2013; Diener, Lucas \& Oishi, 2002) se ha considerado importante asegurar la validez del instrumento utilizado. En este sentido, los análisis factoriales confirmatorios para la escala de satisfacción con la vida (SWLS), atendiendo a criterios estadísticos y sustantivos, muestran que una estructura unifactorial es viable y adecuada con apropiados indicadores de ajuste de fiabilidad y validez.

Con respecto a la predicción de la satisfacción con la vida a través de la autoeficacia en el cuidado de la salud, se han cumplido la mayoría de las hipótesis planteadas a partir del modelo inicial propuesto. Así, las variables contempladas en el modelo alcanzan a explicar el $22 \%$ de la varianza total en la satisfacción con la vida, lo que parece revelar la importancia de la autoeficacia percibida en el cuidado de la salud en la predicción de satisfacción con la vida de las universitarias. Estos resultado corroboran los estudios que han encontrado relación entre la realización de conductas saludables y satisfacción (Sollerhed, et al, 2008, Gunnell, et al, 2014), aportando, este estudio, la evaluación de la creencia que tiene la persona de ser capaz y eficaz en el cuidado de la salud y su valor explicativo en la satisfacción con la vida.

Los diferentes estudios sobre conductas saludables le atribuyen a la actividad física un poder predictivo en la satisfacción con la vida llegando a afirmar que puede explicar el $40 \%$ de la satisfacción (Lyubomirsky, et al, 2005). Por ello, sería esperable haber encontrado una relación directa entre la autoeficacia en la realización del ejercicio físico y la satisfacción con la vida. Sin embargo, en el modelo obtenido se ha encontrado un efecto indirecto, es decir, la autoeficacia en la realización del ejercicio físico predice la satisfacción con la vida, siempre y cuando, la autoeficacia en el cuidado de la alimentación y la autoeficacia en el afrontamiento de problemas estén presentes como mediadores. Se pudiera afirmar en este sentido, la importancia de estudiar, como otras investigaciones han realizado, variables mediadoras (Hecimovich, et al, 2014; León, et al, 2013) para explicar la autoeficacia en la práctica de la actividad física y la satisfacción con la vida.

Los resultados han mostrado una relación directa entre la satisfacción con la vida y la autoeficacia en el afrontamiento de problemas, es decir, si una persona percibe que puede, que es capaz de afrontar de manera eficaz sus problemas, se puede predecir que tendrá mayor satisfacción con la vida. Este hallazgo confirma la importancia que algunos autores le atribuyen al afrontamiento activo de problemas en relación con el bienestar (Sanjuán \& Ávila, 2016; Sanjuán \& Magallares, 2014).

En cuanto a las relaciones indirectas, los resultados indican que la autoeficacia en la evitación del alcohol y el tabaco tiene efectos indirectos en la explicación de la satisfacción con la vida, siendo las variables mediadoras la alimentación y el afrontamiento de problemas. Este hallazgo muestra cierta contradicción con los estudios revisados ya que hubiese sido más esperable que la variable mediadora fuese el ejercicio físico (Amezcua, et al, 2016; Cerkez, et al, 2015) y además, a la luz de algunos estudios, (Annesi \& Tennant, 2014; Teixeira, et al, 2010) sería coherente haber obtenido que la autoeficacia en el cuidado de la alimentación y la práctica del ejercicio físico aparecieran relacionadas entre sí de forma directa. 
En relación a los efectos directos, aparecen entre el ejercicio físico y el afrontamiento de problemas, es decir, la autoeficacia en la práctica del ejercicio físico tiene capacidad predictiva en la autoeficacia en el afrontamiento de problemas. Probablemente este resultado se pueda explicar porque la persona que se siente capaz de hacer ejercicio físico, probablemente lo practicará y este aspecto reducirá el malestar emocional, siendo los beneficios del propio movimiento lo que pudiera favorecer la predisposición a la realización de un afrontamiento activo, sintiéndose la persona más autoeficaz (Reyes- Jarquin \& Hernández- Pozo, 2011; Vallejo- Sánchez \& Pérez- García, 2016). Estos resultados le conceden importancia a la práctica de la actividad física, tal y como tradicionalmente se señala en la literatura (OMS, 2010). Otras dimensiones entre las que se muestran efectos directos son los encontrados entre la autoeficacia en la evitación del tabaco y el alcohol. Estos datos son coherentes con las investigaciones que relacionan ambos consumos (Chacón, et al, 2016).

Se recalca que la autoeficacia en el cuidado de la alimentación y el afrontamiento de problemas son variables que adquieren relevancia: la primera como variable mediadora entre la práctica del ejercicio físico y la evitación de tóxicos (alcohol y tabaco) y la explicación de la satisfacción con la vida; mientras que el afrontamiento de problemas aparece, en los resultados del estudio, tanto como variable mediadora como con capacidad predictiva directa en la satisfacción con la vida. Respecto al cuidado con la alimentación, éste es un aspecto que se trabaja a nivel mundial realizando diversas recomendaciones a la población (Ávila, et al, 2016). Sin embargo, no es el caso del afrontamiento activo ante los problemas, variable que por su relevancia debería trabajarse en la niñez, adolescencia y primera etapa de la adultez (Hills, Dengel \& Lubans, 2015) poniendo especial énfasis en la población universitaria (Chau \& Saravia, 2016) debido, por una parte, al compromiso social que adquieren las universidades (Romero, Cruz, Gallardo \& Peñacoba, 2013), y por otra, al descenso en la práctica de conductas saludables que se produce en estas edades, convirtiendo a este colectivo en población de riesgo (Práxeles, et al, 2016), fundamentalmente en mujeres (Murillo, et al,
2015; Roberts, et al, 2015; Jose, et al, 2011; Castellanos \& Soriano, 2010). En este sentido, y por el mencionado carácter predictivo en la explicación de la satisfacción con la vida, se hace evidente la necesidad de investigar en los procesos asociados a la resolución de problemas en los jóvenes. Además, implementar las estrategias para resolver problemas como una competencia profesional en los distintos estudios universitarios abordándolo de forma específica en programas para la promoción de un estilo de vida activo y saludable. Los jóvenes universitarios constituyen un potencial para el futuro de la sociedad lo que hace que las universidades tengan un compromiso y responsabilidad social apoyando y fomentando la formación de una sociedad más saludable. En este sentido, ya en 1986, la Carta de Ottawa para la promoción de la salud propuso la creación de entornos para fomentar la salud. Así, como entorno saludable, las universidades pueden ofrecer la posibilidad de adquirir, mejorar o mantener el cuidado de la salud entre su población (Romero, et al, 2013). Por ello, algunos autores, señalan que se deben transmitir estrategias para crear y consolidar hábitos de vida saludable y activa (Espejo, et al., 2017) fundamentalmente en la niñez, adolescencia y primera etapa de la adultez (Hills, Dengel \& Lubans, 2015).

Sin embargo, los resultados deben tomarse con cautela al haber realizado el estudio con mujeres estudiantes de Psicología, aspecto que podría sesgar los resultados. Debido a las características muestrales existen limitaciones para extrapolar y generalizar los resultados obtenidos, por tanto, se deben realizar otros estudios con tamaños muestrales mayores y en distintos tipos de instituciones. Además, se necesitan más investigaciones para conocer exactamente qué variables serían las apropiadas para incrementar la autoeficacia en el afrontamiento de problemas. En la misma línea, efectuar estudios comparativos para verificar si, en intervenciones donde se incluya la variable autoeficacia en el afrontamiento de problemas, se obtienen mayores repercusiones en la satisfacción con la vida respecto a intervenciones donde no se incluya dicha variable. También, contrastar las posibles diferencias culturales que podrían existir con universitarios de otros países (GonzálezFuentes \& Andrade, 2016). Del mismo modo, se 
hace absolutamente necesario, conocer otros aspectos implicados en aumentar la satisfacción con la vida de la ciudadanía, y por ende, promover el bienestar, tal y como recomienda la OMS (2013).

\section{Referencias}

Amador, B., Montero, C., Beltrán- Carrillo, V.J., González- Cutre, D., \& Cervelló, E. (2017). Ejercicio físico agudo, agotamiento, calidad del sueño, bienestar psicológico e intención de práctica de actividad física. Revista Iberoamericana de Psicología del Ejercicio y el Deporte, 12(1), 121-127.

Amezcua, M., Hernández, S., Rodríguez, M., \& Quesada, E. (2016). Riesgo ante el consumo colectivo de alcohol entre los jóvenes: Percepciones desde el entorno educativo. Index de Enfermería, 24(1-2), 25-30.

Annesi, J. J., \& Tennant, G.A. (2014). Genralization of theory-base predictions for improved nutrition to adults with morbid obesity: Implications of initiating exercise. International Journal of Clinical and health Psychology, 14, 1-8.

Atienza, F. L., Pons, D., Balaguer, I., \& GarcíaMerita, M. (2000). Propiedades psicométricas de la escala de satisfacción con la vida en adolescentes. Psicothema, 12(2), 314-319.

Ávila, M., Huertas, F. J., \& Tercedor, P. (2016). Programas de intervención para la promoción de hábitos alimentarios y actividad física en escolares españoles de Educación Primaria: Revisión sistemática. Nutrición Hospitalaria, 33(6), 1438-1443. doi:1020960/nh.807

Blanco, H., Ornelas, M., Tristán, J. L., Cocca, A., Mayorga-Vega, D., López-Walle, J., \& Viciana, J. (2013). Editor for creating and applying computerise surveys. Procedia Social and Behavioral Sciences, 106, 935940. doi:10.1016/j.sbspro.2013.12.105

Blanco, J. R., Ornelas, M., Viciana, J., \& Rodríguez-Villalobos, J. M. (2016). Composición factorial de una escala de autoeficacia en el cuidado de la alimentación y salud física en universitarios mexicanos. Nutrición Hospitalaria, 33(2), 379-385.
Castellanos, E., \& Soriano, I. (2010). Sobre la mirada de género en la salud reproductiva y la construcción social de la maternidad. Cuestiones de Género: De la Igualdad y la Diferencia, 5, 89-109.

Cerkez, I., Culjak, Z., Zenic, N., Sekulic, D., \& Kondric, M. (2015). Harmful alcohol drinking among adolescents: The influence of sport participation, relilgiosity and parental factors. Journal of Child and Adolescents Substance Abuse, 24(2), 94-101.

Chacón, R., Castro, M., Caracuel, R., Padial, R. Collado, D., \& Zurita, F. (2016). Perfiles de consumo de alcohol y tabaco en adolescentes. Health and Addicitons, 16(2), 93-104.

Chau, C., \& Saravia, J.C. (2016). Conductas de salud en estudiantes universitarios limeños: Validación del CEUJU. Revista Iberoamericana de Diagnóstico y Evaluación - e Avaliação Psicológica, 41(1), 90-103

Diener, E., Emmons, R. A., Larsen, R. J., \& Griffin, S. (1985). The satisfaction with life scale. Journal of Personality Assessment, 49(1), 71-75.

Diener, E., Lucas, R., \& Oishi, S. (2002). Subjetive well-being: The science of happiness and life satisfaction. In C.Snyder y S. López (Eds), Handbook of positive psychology (pp.63-73). New York: Oxford University Press.

Espejo, T., Martínez, A., Chacón, R., Zurita, F., Castro, M., \& Cachón, J. (2017). Consumo de alcohol y actividad física en adolescentes de entorno rural. Health and Additions, 17(1), 97-105.

González- Cantero, J. O., Oropeza, R., Padrós, F., Colunga, C., Montes, R., \& GonzálezBecerra V.H. (2017). Capital psicológico y su relación con el estilo de vida de universitarios mexicanos. Nutrición Hospitalaria, 34(2), 439-443.

González- Fuentes, M. B., \& Andrade, P. (2016). Escala de bienestar psicológico para adolescentes. Revista Iberoamericana de Diagnóstico y Evaluación - e Avaliação Psicológica, 42(2), 69-83.

Gunnell, K.E., Crocker, P.R., Mack, D.E., Wilson, P.M., \& Zumbo, B.D. (2014). Goal contents, motivation, psychological need satisfaction, well- being and physical activity: A test of 
self-determination theory over 6 months. Psychology of Sport and Exercise, 15, 19-29. doi:10.1016/j.psychsport.2013.08.005

Hecimovich, M. D., Peiffer, J. J., \& Harbaugh, A. G. (2014). Development and psychometric evaluation of a post exercise exhaustion scale utilising the Rasch measurement model. Psychology of Sport and Exercise, 15, 569579. doi:10.1016/j.psychsport.2014.06.003

Hills, A. P., Dengel, D. R., \& Lubans, D. R. (2015). Supporting public health priorities: Recomendations for psysican education and physical activity promotion in schools. Progress in Cardiovascular Diseases, 57(4), 368-374. doi:10.1016/j.pcad.2014.09.010

Jose, K. A., Blizzard, L., Dwyer, T., Mckercher, C., \& Venn, A. J. (2011). Childhood and adolescent predictors of leisuse time physical activity during the transition from adolescente to adulthood: A population base cohort study. International Journal of Behavioral Nutrition and Psysical Activity, 8(54).

doi:10.1186/1479-5868-8-54

León, J., Núñez, J. L., Domínguez, E. G., \& Martín-Albo, J. (2013). Motivación intrínseca, autoconcepto físico y satisfacción con la vida en practicantes de ejercicio físico: Análisis en un modelo de ecuaciones estructurales en el entorno de programación R. Revista Iberoamericana de Psicología del Ejercicio y el Deporte, 8(1), 39-58.

Losada, F., Varela, J., \& Lévy J. P (2006). Modelización con estructuras de covarianzas en ciencias sociales. A Coruña: Netbiblo.

Lyubomirsky, S., Sheldon, K., \& Sihkade, D. (2005). Pursuing happiness: The architecture of sustainable change. Review of General Psychology, 9, 111-131. doi:10.1037/1085-2680.9.2.111

Luszczynska, A., \& Schwarzer, R. (2005). Social congnitive theory. In M. Conner y P. Norman (Eds), Predicting health behaviour $\left(2^{\circ} \mathrm{ed}, \mathrm{pp}\right.$. 127-169). Buckingham Open University Press.

Maddux, J. E. (2005). Self-efficacy. In C.R. Snyder, \& S. J. López (Eds), Handbook of positive psychology (pp. 277-287). Oxford: Oxford University Press.

Martín-Payo, R., Suárez-Álvarez, Jo, Ameva, M. E., Duaso, M. J., \& Álvarez, E. (2016).
Adherence to healthy diet and physical activity in clinical patients. Psicothema, 28(4), 457-464. doi:10.7334/psicothema.2016.227

Murillo, B., García, E., Aibar, A., Julian, J. A., García-González, L. Martín-Albo, J., \& Estrada, S. (2015). Factors associated with compliance with psysical activity recommendations among adolescents in Huesca. Revista de Psicología del Deporte, 24(1), 147-154.

Organización Mundial de la Salud (2010). Recomendaciones mundiales sobre la actividad física para la salud. Ginebra: La Organización.

Organización Mundial de la Salud (2013). The European health report 2012: Charting the way to well- being. Copenhague: WHO.

Oros, L. B. (2017). Valores normativos de la Escala multidimensional de autoeficacia infantil para población Argentina. Revista Iberoamericana de Diagnóstico y Evaluación - e Avaliação Psicológica, 44(2), 172-181. doi:10.21865/RIDEP 44.2.14

Povey, R., Conner, M., Sparks, P., \& James, R., \& Shepherd, R. (2000). Application of the therapy behaviour theory to two dietary behaviours: Roles of perceived control and self-efficaciy. British Journal of Health Psychology, 5(2), 21-139.

Práxeles, A., Sevil, J., Moreno, A., del Villar, F., \& García-Conzález, L. (2016). Niveles de actividad física en estudiantes universitarios: Diferencias en función del género, la edad y los estados de cambio. Revista Iberoamericana de Psicología del Ejercicio y el Deporte, 11(1), 123-132.

Reyes-Jarquin, K., \& Hernández-Pozo, M. R. (2011). Análisis crítico de los estudios que exploran la autoeficacia y bienestar vinculados al comportamiento saludable. Journal of Behavior, Health \& Social Issues, 3(2), 5-24. doi:10.5460/jbhsi.v3.3.29915

Roberts, S., Reeves, M., \& Ryrie, A. (2014). The influence of physical activity, sport and exercise motives among UK- based university students. Journal of Further and Higher Education. doi:10.1080/0309877X-2014.938265

Romero, A., Cruz, S., Gallardo, C., \& Peñacoba, C. (2013). Cómo promover la salud y el bienestar en la comunidad universitaria. 
Revista Iberoamericana de Psicología y Salud, 4(2), 49-68.

Salanova, M., \& Martínez, J. M., Bresó, E., Llorens, S., \& Grau, R. (2005). Bienestar psicológico en estudiantes universitarios. Facilitadores y obstaculizadores del desempeño académico. Anales de Psicología, 21, 170-180.

Sanjuán, P., \& Ávila, M. (2016). Afrontamiento y motivación como predictores de bienestar subjetivo y psicológico. Revista de Psicopatología y Psicología Clínica, 21, 1-10. doi:10.5944/rppc.vol21.num1.2016.15401

Sanjuán, P., \& Magallares, A. (2014). Coping strategies as mediating variables between selfserving atttibutional bias and subjective wellbeing. Journal of Happiness Studies, 15, 443453. doi:10.1007/s/0902-013-9430-2

Sollerhed, A. C., Apitzsch, E., Rastam, L., \& Ejlertsson, G. (2008). Factors associated with Young children's self-perceived physical competence and self-reported physical activity. Health Education Research, 23, 125136. doi:10.1093/her/cym010

Teixeira, P. J., Silva, M. N., Coutinho, S. R., Palmeira, A. L., Mata, J., Viera, P. N., Carracas, E. V., Santos, T. C., \& Sandinha, L. B. (2010). Mediators of weight loss and weight loss maintenance in middle- aged women. Obesity, 18, 725-735.

Thompson, B. (2004). Exploratory and Confirmatory Factor Analysis. Understanding concepts and applications.. Washington, D C: American Psychological Association.

Thrasher, J. F., Swayampakala, K., Borland, R., Nagelhout, G, Young, H., Hammond, D., Bansal-Travers, M., Thompson, M., \& Hardin, J. (2016). Influence of self- efficacy, and reactance on responses to cigarette health warnings: a longitudinal study of adult smokers in Australia and Canada. Health Communication, 31(12), 1517-1526. doi:10.1018/10410236.2015.1089456

Vallejo-Sánchez, B., \& Pérez- García, A. M. (2016). Estrés vital: Variables psicológicas y sociodemográficas predictoras del maestro emocional. Accion Psicológica, 13(1), 159178. 